\title{
Topological Correction of Brain Surface Meshes Using Spherical Harmonics
}

\author{
Rachel Aine Yotter, Robert Dahnke, and Christian Gaser \\ Friedrich-Schiller University, Department of Psychiatry, Jahnstr. 3, \\ 07745 Jena, Germany \\ \{Rachel.Yotter, Robert.Dahnke, Christian. Gaser\} @uni-jena.de
}

\begin{abstract}
A brain surface reconstruction allows advanced analysis of structural and functional brain data that is not possible using volumetric data alone. However, the generation of a brain surface mesh from MRI data often introduces topological defects and artifacts that must be corrected. We show that it is possible to accurately correct these errors using spherical harmonics. Our results clearly demonstrate that brain surface meshes reconstructed using spherical harmonics are free from topological defects and large artifacts that were present in the uncorrected brain surface. Visual inspection reveals that the corrected surfaces are of very high quality. The spherical harmonic surfaces are also quantitatively validated by comparing the surfaces to an "ideal" brain based on a manually corrected average of twelve scans of the same subject. In conclusion, the spherical harmonics approach is a direct, computationally fast method to correct topological errors.
\end{abstract}

\section{Introduction}

In brain analyses, it is sometimes desirable to deform the grey matter (GM) sheet into a sphere. A brain surface is not required to be homeomorphic with a sphere (e.g., contain no topological errors) before being mapped to a sphere. However, correcting topological defects is a necessary prerequisite for inter-subject analysis. When analyzing inter-subject data, a common coordinate system must be used to extract meaningful comparisons between the subjects. Since a brain surface mesh is roughly spherical, a logical choice is a spherical coordinate system.

There are three common representations of the cortical surface: the interface between GM and white matter (WM); the interface between GM and cerebrospinal fluid (CSF); and the central surface (CS), which is approximately midway between the GM/WM and GM/CSF interfaces. Compared to the other representations, the CS provides an inherently less distorted representation of the cortical surface [1].

Due to noise, partial volume effects, and other problems during the MRI data acquisition process, a brain surface mesh reconstructed from volumetric data often contains topological defects and artifacts. Topological defects can include handles and holes that prevent the surface from being homeomorphic with a sphere. Artifacts are topologically correct sharp peaks (usually due to noise) that have no relation to brain anatomy. Both types of errors should be repaired before the surface is inflated to a 
sphere. Artifacts can be minimized via smoothing. However, accurately correcting topology defects is a more complicated endeavor.

Here, we propose to use spherical harmonics for the first time to accurately correct topological defects. Spherical harmonic analysis has recently been applied to brain surface meshes, usually in the realm of shape analysis [2]. It has been applied to quantifying structural differences in subcortical structures [3-6] and full surfaces [7, 8]. Spherical harmonic analysis can be described as a Fourier transform on a sphere that decomposes the brain surface data into frequency components. The surface can also be reconstructed from the harmonic information. Generally, the RMS error of the reconstructed surface decreases as the number of coefficients increases. Furthermore, the coefficients can be weighted to achieve a smoothing effect [9].

A drawback to spherical harmonics is the computation time required to calculate the coefficients. However, a modification of the fast Fourier transform compatible with spherical coordinates significantly decreases the computation time, since it is no longer necessary to directly calculate the coefficients $[10,11]$.

Previously, there were two general approaches used to correct topology defects. The first approach is to start with a surface with the desired topology and deform it to match the brain surface. This includes active contours or deformable models, and there is a wealth of literature on variations of this method applied to brain data; for a review, see $[12,13]$. The second approach is to retrospectively correct the topology after the brain data has been segmented into WM and GM [14-19].

In this paper, we show for the first time that spherical harmonics processing can accurately and quickly repair topological defects. The spherical harmonics approach retrospectively corrects topological errors directly on the brain surface mesh, and results in an accurately reconstructed cortical surface free from topological defects and large artifacts.

\section{Methods}

Topological correction using spherical harmonics can be subdivided into four processing steps. First, we resample a spherical mapping of the surface mesh and calculate coordinates of location for each sampled point based on its approximate location in the surface mesh. For this step, a spherical mapping of the cortical surface is required. This spherical mapping is not homeomorphic with a sphere, since it still contains the topological defects from the original surface. However, low distortion in the spherical mapping improves the re-parameterization process.

Second, the coordinates of location mapped on the regularly sampled sphere are forward transformed using a Fourier transform to extract the harmonic content. The harmonic content is either left intact or bandwidth-limited using a low-pass Butterworth filter. This creates two sets of data that are passed through a third step, namely an inverse Fourier transform that reconstructs the harmonic data into two distinct surfaces: a high-bandwidth surface and a bandwidth-limited smoothed surface. In the high-bandwidth reconstruction, the topological defects are replaced with a spiked topology. Finally, the spiked topology is corrected by replacing local patches with points from a lower-bandwidth reconstructed surface. The result is a defect-free brain surface that retains a high level of detail and replaces the topological defects with a smoothed, more anatomically accurate representation. 


\subsection{Spherical Parameterization and Generation of Tensor Fields}

To analyze the harmonic content of a spherical surface, the spherical surface needs to have regularly sampled points with respect to $\theta$ and $\phi$, where $\theta$ is the co-latitude and $\phi$ is the azimuthal coordinate. However, spherical maps of cortical surfaces usually do not have regularly sampled points, so an initial step is a re-parameterization of the spherical surface. In order to accomplish this, points are generated from equally sampled values of $\theta$ and $\phi$ for all members in the sets, such that there are $2 B$ points per set, where $B$ is the bandwidth. For each regularly sampled spherical point, the intersecting polygon on the cortical spherical mapping is found. Within that intersecting polygon, a coordinate of location is approximated using barycentric coordinates, e.g., the location of the regularly sampled point within the intersecting polygon on the spherical mapping determines a certain set of barycentric coordinates, and these barycentric coordinates are then used within the corresponding polygon in the original cortical surface to find the cortical location. The result is a regularly sampled spherical map in which every point is associated with a coordinate related to the location on the original cortical surface.

\subsection{Harmonic Analysis}

The harmonic content of a spherical mesh can be obtained using normalized spherical harmonics $Y_{l}^{m}(\theta, \phi)$ :

$$
Y_{l}^{m}(\theta, \phi)=P_{l}^{m}(\cos \theta) e^{\mathrm{i} m \phi},
$$

where $l$ and $m$ are integers with $|m| \leq l$, and $P_{l}^{m}$ is the associated Legendre function defined by:

$$
P_{l}^{m}(x)=\frac{1}{2^{l} l !}\left(1-x^{2}\right)^{\frac{m}{2}} \frac{d^{l+m}}{d x^{l+m}}\left(x^{2}-1\right)^{l} .
$$

A square-integrable function $f(\theta, \phi)$ on the sphere can be expanded in the spherical harmonic basis such that:

$$
f(\theta, \phi)=\sum_{l=0}^{B} \sum_{m=-l}^{l}\left\|Y_{l}^{m}\right\|_{2}^{-2} \hat{f}(l, m) \cdot Y_{l}^{m},
$$

where the coefficients $\hat{f}(l, m)$ are defined by the inner product $\hat{f}(l, m)=\left\langle f, Y_{l}^{m}\right\rangle$ and the L2-norm of $Y_{l}^{m}$ is given by:

$$
\left\|Y_{l}^{m}\right\|_{2}^{-2}=\frac{4 \pi}{2 l+1} \cdot \frac{(l+m) !}{(l-m) !} .
$$

It is possible to solve this system directly by finding the bases first, but a more efficient approach is to use a divide-and-conquer scheme as described in [10].

The calculated coefficients are either left as is or are bandwidth-limited using a 128-order Butterworth low-pass filter in order to exclude the contributions from 
higher-frequency coefficients. A Butterworth filter reduces ringing artifacts and results in a smoother reconstructed surface. Finally, the harmonic content is processed through an inverse Fourier transform to produce bandwidth-limited coordinates associated with each spherical point. These filtered coordinates are used to reconstruct a cortical mesh without topological defects.

\subsection{Refinement of Reconstructed Cortical Meshes}

After reconstruction via spherical harmonics, the resulting cortical mesh is homeomorphic with a sphere. However, adjacent to regions formerly containing topological defects, the spherical harmonics reconstructed surface generally replaces the defect with a spiked topology that does not correspond to actual brain anatomy (Figure 1). The spiked appearance is only present if the bandwidth is high enough to admit higher frequencies; if a lower bandwidth is used, the surface near former topological defects is smooth and well-reconstructed, yet some detail is also lost.
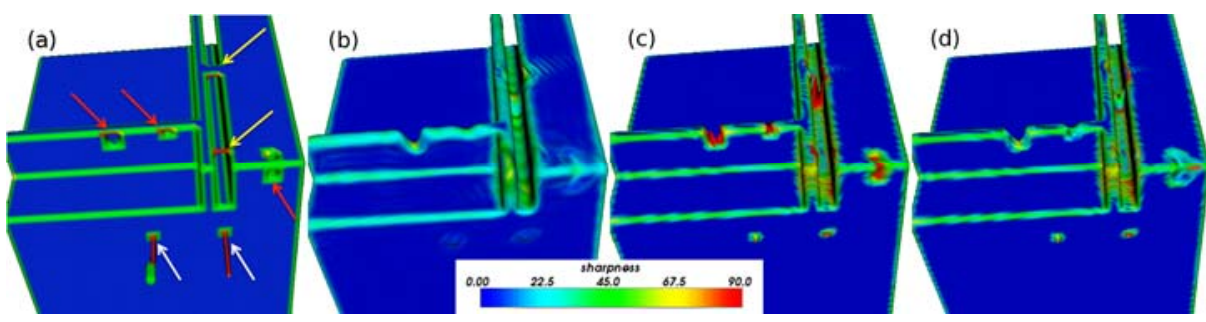

Fig. 1. Topological correction using spherical harmonics relies on a union between a highbandwidth $(B=1024)$ and bandwidth-limited $\left(B_{l}=64\right)$ surface. A cubic surface (a) contains three holes (red arrows), two bridges (yellow arrows), and two artifacts (white arrows). The bandwidth-limited surface (b) corrects the topological errors but loses detail. The highbandwidth reconstruction (c) replaces topological defects with sharp spikes. The union of the two surfaces (d) optimizes detail retention and topological error correction. Generally, smaller topological defects are repaired more accurately than larger topological defects.

As a final step, these surfaces are combined such that the regions that exhibit spiked topology in the higher-bandwidth $(B=1024)$ surface are replaced with patches from a bandwidth-limited $\left(B_{l}=64\right)$ cortical reconstruction. The lower bandwidth was chosen such that the spiked regions were smooth but the surface retained the approximate shape of the original cortical surface, such that the union of the two surfaces does not result in large discontinuities.

Topologically spiked regions are found by calculating a sharpness value for each point. The sharpness is the maximum angle between normals of nearest-neighbor polygons. If sharpness is above a threshold, then this point and a set of neighbors are replaced with corresponding points in the bandwidth-limited cortical surface.

Theoretically, optimal defect correction in both the WM and CS should result in the same sharpness threshold, since the bandwidths of the two proposed surfaces are the same. Basing the sharpness threshold on a certain standard deviation within the sharpness histogram is not adequate, since the brain surfaces vary widely in the number and size of their topology defects. Instead, the sharpness threshold should be set to 
maximize the overlap between the proposed patch area and the defect regions, while minimizing patches outside of the defect regions. It was found that a threshold of $t_{s}=$ $110^{\circ}$ fulfilled these criteria, and separate threshold optimization of the WM and CS surfaces resulted in the same threshold value.

By default, the patch includes the high-sharpness point and the two nearest neighbors. However, it is possible that there may be a discontinuity between the patch and the high-bandwidth surface at the edge. Our approach was to measure the distance between the points surrounding the patch and their corresponding points in the high-bandwidth surface, and to include in the patch any points that were more than 2 $\mathrm{mm}$ distant. The union of these two surfaces results in a continuous cortical surface that retains high-frequency information for gyri and sulci, and an anatomically accurate reconstruction of regions that previously contained topological defects.

\subsection{Sample Data Set and Verification of Results}

Alongside visual validation, we wished to quantitatively assess the validity of the corrected surface. Our approach was to create an "ideal" brain by averaging twelve scans of the same brain. The averaging reduces noise and almost all of the topological defects. The remaining defects were extremely small and corrected manually using publicly available manual editing tools.

The sample data set included 12 brain scans of the same brain. These scans were acquired with a $1 \mathrm{~mm}$ isotropic resolution on two $1.5 \mathrm{~T}$ scanners. Each scan was processed to produce WM and CS surface representations for each hemisphere using publicly available software [20,21]. The CS spherical mappings were additionally postprocessed using in-house tools to improve re-parameterization accuracy.

All topology corrected brain surfaces were then compared to the "ideal" averaged brain surface using mean distance error and outlier reduction percent. The mean distance error $d_{e}$ is the average minimum distance between a set of points $X$ and a surface $S$. The minimum distance function $d(p, S)$ between a point $p \in X$ and the surface $S$ can be defined as:

$$
d(p, X)=\min _{p^{\prime} \in S}\left\|p-p^{\prime}\right\|,
$$

where $p^{\prime}$ is a point on surface $S$. The mean distance error is then defined as follows:

$$
d_{e}=\frac{1}{N_{p}} \sum_{p \in X} d(p, S),
$$

where $N_{p}$ is the number of points in the set of points $X$.

Because the distance error histograms are not significantly different, a new metric was developed to quantify the reduction in distance error. The outlier reduction percent represents the fraction of points that remain above a distance error threshold set in the uncorrected brain surface, such that:

$$
O P=\left(1-\frac{N_{t}}{N_{t}^{o}} \cdot \frac{N_{p}^{o}}{N_{p}}\right) \times 100,
$$


where $N_{t}^{\circ}$ and $N_{t}$ are the number of points whose distance errors are above threshold in the original brain surface and the corrected surface, respectively, and $N_{p}{ }^{\circ}$ and $N_{p}$ are the total number of mesh points in the original brain surface and the corrected surface, respectively. The threshold is set to include the top 5\% points with the largest distance error in the uncorrected brain surface. A value of $100 \%$ indicates that all outliers have been removed, while a value of $0 \%$ indicates no improvement.

\section{Results and Discussion}

Visual inspection reveals that the surface reconstructed using spherical harmonics is free from topological defects and is similar to the "ideal" averaged brain surface (Figure 2). The approach is valid for both the central surface and the WM surface. High-bandwidth spherical harmonic reconstruction replaces topological defects with a spiked topology (Figure 2b,f). These regions are repaired by replacing local patches with points from a bandwidth-limited reconstruction (Figure 2c,g). The result is a corrected surface that is closer to the "ideal" averaged surface compared to the original uncorrected surface (Figure 2d,h).

Quantitatively, spherical harmonics generates a corrected surface that has a lower mean distance error $(0.5371$ for WM; 0.6127 for $\mathrm{CS})$ compared to the uncorrected surface $(0.7485$ for WM; 0.7757 for CS), when both surfaces are compared to the ideal surface. The outlier reduction percent was $85 \%$ for $\mathrm{WM}$ and $50 \%$ for CS, indicating that points with large errors are significantly reduced.

There is almost no difference between the spherical harmonic surface and the original uncorrected surface in areas that do not contain topological defects or artifacts (Figure 3). It is mostly the corrections in the areas of topological defects that are responsible for the improved distance error metrics.

By using a fast Fourier transformation rather than calculating the spherical harmonic coefficients directly, topology correction using spherical harmonics requires

(a)
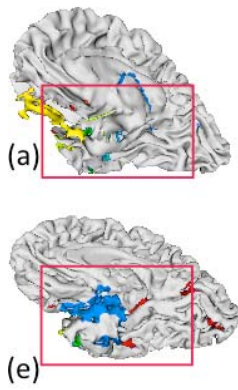

(b)

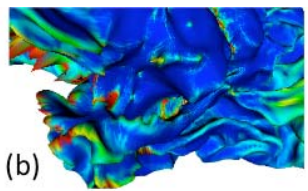

(f)

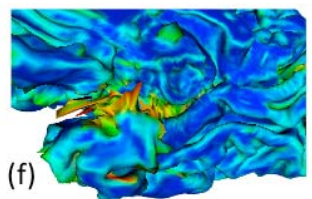

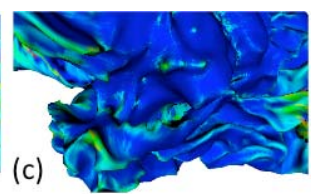
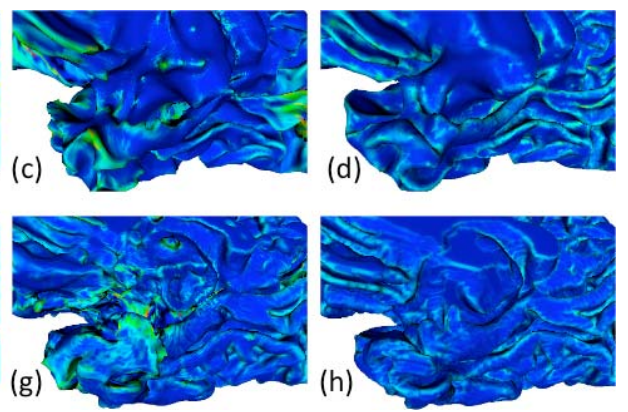

Fig. 2. Spherical harmonic correction of the WM (a-d) and CS (e-h). The original surfaces (a, e) contain holes, handles, and large artifacts (marked for visibility). These are eliminated via spherical harmonics reconstruction. At high bandwidths $(b, f)$, the topological errors are replaced by a spiked topology. By patching these regions, the surface is reconstructed consistent with cortical anatomy $(c, g)$. As a reference, the "ideal" brain surface is shown in (d, h). In (b-d) and (f-h), the colors represent sharpness, with red indicating high sharpness. 

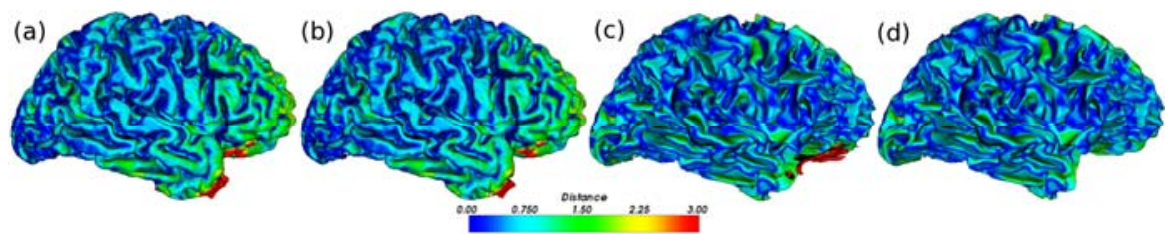

Fig. 3. Away from topological defects, surface information remains unchanged. The uncorrected surfaces for CS (a) and WM (c) are almost identical to the spherical harmonics corrected surfaces for CS (b) and WM (d), except around regions near topological defects (red). The distance in $\mathrm{mm}$ is the error between these surfaces and the "ideal" averaged surface.

approximately 5 minutes on a $2.4 \mathrm{GHz}$ iMac for a mesh that contains 150,000 vertices. The resulting mesh contains approximately the same number of vertices.

These results are the raw output from the spherical harmonic reconstruction. There is no post-processing beyond patching the bandwidth-limited reconstruction into the high-bandwidth surface. Reconstruction can be improved by increasing the accuracy of the initial segmentation or via smoothing. Either the full brain or local regions containing high curvature or high sharpness would benefit from smoothing, especially for the CS with its low curvature. The WM surface can be further optimized using a post-refinement process that uses the original $\mathrm{T} 1$ data in the correction routines.

\section{Conclusion}

The spherical harmonics approach is a fast, straightforward method to accurately correct topological defects. We have applied spherical harmonics to cortical surfaces with the goal of correcting topological defects and artifacts, and we have shown that this approach accurately reconstructs the cortical surface. The resulting surfaces have lower mean distance errors to an "ideal" template than the original uncorrected surfaces. The computation time per hemisphere is on the order of a few minutes, suggesting that spherical harmonic reconstruction is a fast, accurate approach for topology error correction. It is highly suitable for inclusion into a processing pipeline for cortical surface analysis.

Acknowledgments. This work was supported by the following grants: BMBF 01EV0709 and BMBF 01GW0740.

\section{References}

1. Van Essen, D.C., Maunsell, J.H.R.: Two-dimensional maps of the cerebral cortex. Journal of Comparative Neurology 191(2), 255-281 (1980)

2. Brechbühler, C., Gerig, G., Kübler, O.: Parametrization of closed surfaces for 3-D shape description. Computer Vision and Image Understanding 61(2), 154-170 (1995)

3. Kelemen, A., Szekely, G., Gerig, G.: Elastic model-based segmentation of 3-D neuroradiological data sets. IEEE Transactions on Medical Imaging 18(10), 828-839 (1999)

4. Gerig, G., Styner, M., Jones, D., Weinberger, D., Lieberman, J.: Shape analysis of brain ventricles using SPHARM. In: MMBIA 2001, Kauai, HI, USA, pp. 171-178 (2001) 
5. Shenton, M.E., Gerig, G., McCarley, R.W., SzÈkely, G.B., Kikinis, R.: Amygdalahippocampal shape differences in schizophrenia: the application of 3D shape models to volumetric MR data. Psychiatric Research: Neuroimaging 115(1-2), 15-35 (2002)

6. Styner, M., Lieberman, J.A., Pantazis, D., Gerig, G.: Boundary and medial shape analysis of the hippocampus in schizophrenia. Medical Image Analysis 8(3), 197-203 (2004)

7. Shen, L., Chung, M.K.: Large-scale modeling of parametric surfaces using spherical harmonics. In: 3DPVT 2006, Chapel Hill, NC, pp. 294-301 (2006)

8. Chung, M.K., Shen, L., Dalton, K.M., Davidson, R.J.: Multi-scale voxel-based morphometry via weighted spherical harmonic representation. In: Yang, G.-Z., Jiang, T.-Z., Shen, D., Gu, L., Yang, J. (eds.) MIAR 2006. LNCS, vol. 4091, pp. 36-43. Springer, Heidelberg (2006)

9. Chung, M.K., Dalton, K.M., Li, S., Evans, A.C., Davidson, R.J.: Weighted Fourier series representation and its application to quantifying the amount of gray matter. IEEE Transactions on Medical Imaging 26(4), 566-581 (2007)

10. Healy, D.M., Rockmore, D.N., Moore, S.S.B.: FFTs for the 2-sphere-improvements and variations. Technical Report, Dartmouth College (1996)

11. Kostelec, P.J., Maslen, D.K., Healy, D.M., Rockmore, D.N.: Computational harmonic analysis for tensor fields on the two-sphere. J. Comp. Physics 162(2), 514-535 (2000)

12. McInerney, T., Terzopoulos, D.: Deformable models in medical image analysis: a survey. Med. Image Anal. 1, 91-108 (1996)

13. Montagnat, J., Delingette, H., Ayache, N.: A review of deformable surfaces: topology, geometry and deformation. Image Vis. Comp. 19, 1023-1040 (2001)

14. Fischl, B., Liu, A., Dale, A.M.: Automated manifold surgery: constructing geometrically accurate and topologically correct models of the human cerebral cortex. IEEE Trans. Med. Imaging 20, 70-80 (2001)

15. Shattuck, D.W., Leahy, R.M.: Automated graph-based analysis and correction of cortical volume topology. IEEE Trans. Med. Imaging 20, 1167-1177 (2001)

16. Han, X., Pham, D.L., Tosun, D., Rettmann, M.E., Xu, C., Prince, J.L.: CRUISE: Cortical reconstruction using implicit surface evolution. Neuroimage 23, 997-1012 (2004)

17. Jaume, S., Rondao, P., Macq, B.: Open Topology: A Toolkit for Brain Isosurface Correction. In: MICCAI (2005)

18. Segonne, F., Pacheco, J., Fischl, B.: Geometrically Accurate Topology-Correction of Cortical Surfaces Using Nonseparating Loops. IEEE Trans. Med. Imag. 26, 518-529 (2007)

19. Wood, Z., Hoppe, H., Desbrun, M., Schroder, P.: Removing excess topology from isosurfaces. ACM Trans. Graph. 23, 190-208 (2004)

20. Drury, H.A., Van Essen, D.C., Anderson, C.H., Lee, C.W., Coogan, T.A., Lewis, J.W.: Computerized mappings of the cerebral cortex: A multiresolution flattening method and a surface-based coordinate system. Journal of Cognitive Neuroscience 8(1), 1-28 (1996)

21. Fischl, B., Sereno, M.I., Dale, A.M.: Cortical surface-based analysis: II: Inflation, flattening, and a surface-based coordinate system. Neuroimage 9(2), 195-207 (1999) 